Annuaire suisse de politique de développement

6 | 1987

Annuaire Suisse - Tiers Monde 1986

\title{
Nicaragua : la banane... avec la petite différence
}

\author{
Ueli Tecklenburg
}

\section{OpenEdition}

\section{Journals}

Édition électronique

URL : http://journals.openedition.org/aspd/1235

DOI : $10.4000 /$ aspd.1235

ISSN : 1663-9669

\section{Éditeur}

Institut de hautes études internationales et du développement

\section{Édition imprimée}

Date de publication : 1 janvier 1987

Pagination : 183-189

ISSN : 1660-5934

\section{Référence électronique}

Ueli Tecklenburg, « Nicaragua : la banane... avec la petite différence », Annuaire suisse de politique de développement [En ligne], 6 | 1987, mis en ligne le 06 mars 2013, consulté le 08 septembre 2020. URL http://journals.openedition.org/aspd/1235 ; DOI : https://doi.org/10.4000/aspd.1235 


\title{
NICARAGUA : LA BANANE... AVEC LA PETITE DIFFERENCE
}

\author{
Ueli Tecklenburg
}

Au début, il y a un petit entrefilet dans le journal «Le Monde», en juin 1985. Celui-ci annonce que, pour la première fois en Europe, un bateau bananier nicaraguayen est en train de décharger sa cargaison dans le port de Gand en Belgique, un mois à peine après le décret d'embargo commercial contre le Nicaragua promulgué par le président Reagan.

Cette information met "la puce à l'oreille» de quelques tiers-mondistes, liés au mouvement du "commerce plus juste» et convaincus qu'il faut réagir au défi lancé par l'administration Reagan. Dès le début, ces militants défendent l'idée que la poursuite des relations économiques et de coopération avec le Nicaragua est un facteur de développement et de paix et que l'étranglement économique, poussant le régime sandiniste dans ses derniers retranchements, met les libertés individuelles beaucoup plus en danger que l'ouverture commerciale.

D'autres raisons plaident en faveur d'une action bananes du Nicaragua: depuis les essais infructueux de la Comunbana, la société exportatrice commune à plusieurs pays latino-américains, c'est la première fois qu'un pays exportateur de bananes tente de maîtriser la filière d'exportation, d'habitude chasse gardée des multinationales bananières. Ce contrôle de la filière, en aval de la production, est un élément important d'une stratégie de développement autonome et, à ce titre, mérite l'attention des milieux tiers-mondistes européens. D'autre part, et même si la pratiqũe sandiniste peut prêter le flanc à la critique, il est indéniable que les conditions de travail et de vie des ouvriers des plantations de bananes au Nicaragua se sont nettement améliorées: engagements fixes, salaires s'alignant sur ceux de la moyenne des autres exportateurs centre-américains, services sociaux gratuits. Tous les observateurs ayant visité les plantations ont aussi constaté une motivation plus grande des travailleurs, une volonté manifeste de participer à la reconstruction du pays. En Suisse, enfin, le contexte public de l' «Appel pour un commerce équitable», en voie de préparation à cette époque, offre une bonne opportunité d'illustrer concrètement, par l'exemple d'un produit, ce que peut être un tel commerce.

\section{Des bananes dans le commerce alternatif?}

$\mathrm{Vu}$ les structures bénévoles de distribution du commerce alternatif notamment des Magasins du Monde - , il est difficile d'envisager d'importer un produit frais tel que la banane.

Toujours en juin 1985, des représentants d'OS3 (Organisation Suisse - 
Tiers Monde) (1) participent à une réunion des ATO' européens (Alternative Trade Organization: organisations telles qu'OS3 dans d'autres pays européens) où ils apprennent avec étonnement que les Magasins du Monde belges se sont déjà lancés avec un succès considérable dans "l'aventure bananes». A partir de là, l'idée de la vente des bananes Nica en Suisse commence à prendre forme. Le comité d'OS3 est alors saisi et il se prononce en faveur du principe, tout en restant sceptique sur les possibilités d'une vente continue par le secteur alternatif en Suisse.

Très rapidement, des contacts sont établis avec le groupe «Bananenfrauen», organisation suisse alemanique qui dispose de larges connaissances dans le domaine du marché bananier. Les participantes de ce groupe restent également très sceptiques sur les possibilités et même l'utilité d'une distribution des bananes Nica par les canaux du commerce tiersmondiste: à juste titre, elles objectent qu'une introduction de cette banane en Suisse n'a sens que dans la mesure où le commerce traditionnel s'en empare, le secteur alternatif - avec tout ce qu'il peut apporter pour sensibiliser l'opinion publique - ne pouvant jouer que le rôle de médiateur.

Le commerce traditionnel de fruits et légumes est donc contacté; les grandes chaînes de distribution, importateurs fruitiers indépendants: en vain! La banane du Nicaragua n'a pas la cote sur le marché suisse. Le plus souvent, sa prétendue mauvaise qualité est avancée pour justifier le refus.

\section{Le Nicaragua et ses bananes}

Concernant le marché bananier, il convient peut-être de rappeler les difficultés auxquelles le Nicaragua s'est vu confronté avec le boycott commercial américain.

Jusqu'en 1982, la Standard Fruit Co (une des trois plus grandes multinationales bananières) a pris en charge la production et la commercialisation des bananes (commerce qu'elle avait repris dix ans auparavant à la United Brands $\mathrm{Co}$ ), après avoir passé une convention de commercialisation avec les sandinistes après la révolution de 1979. La production bananière et l'infrastructure d'exportation étaient orientées vers I'Océan Pacifique. La Standard Fruit acheminait la plupart des bananes vers la Californie. Du jour au lendemain, la multinationale fruitière dénonça le contrat la liant au gouvernement de Managua et abandonna les plantations nicaraguayennes. Dès lors, les sandinistes furent obligés de reprendre la gestion de la production bananière et de trouver de nouveaux débouchés commerciaux. Ils les trouvèrent par le biais d'une autre entreprise américaine: la Pandol Brothers Co qui a son siège en Californie. Tout continuait donc de se passer du côté Pacifique.

L'embargo de mai 1985 met un terme abrupt à ce commerce et les Nicaraguayens - qui en saine prévision des événements, avaient déjà établi des contacts - se voient obligés de se tourner vers I'Europe (2). Ce choix comporte un inconvenient majeur: les temps de transport - jusqu'alors de trois à quatre jours pour la Californie - s'élèvent à dix, voire quinze jours pour 
l'Europe. Dans le cas de la banane, cette différence est cruciale. Pour éviter le pourrissement, les bananes doivent être coupées à un autre stade de mûrissement et être traitées d'une autre manière. Les Nicaraguayens n'y sont malheureusement pas préparés et il semblerait que le premier arrivage en Belgique fut une catastrophe du point de vue de la qualité du fruit.

\section{Les tiers-mondistes comme promoteurs commerciaux}

En automne 1985, les importateurs suisses font un blocage quasi total. Pour quelles raisons? II est difficile de vérifier si des considérations d'ordre politique sont à l'origine de ce refus. II semble plutôt qu'un autre élément, d'ordre commercial, a joué un rôle déterminant. Le commerce de la banane étant dominé en Suisse par les Chiquitas et les Dôles, l'on a peine à croire que les tiers-mondistes sont capables d'ouvrir la brèche dans ce marché.

Pour les "promoteurs tiers-mondistes» de la banane Nica, il ne reste à ce moment qu'une seule issue pour la réalisation de ce marché : une action ponctuelle avec des bananes mûries en Belgique et importées par leurs propres moyens. Un défi, car personne dans le commerce alternatif n'a la moindre expérience dans l'importation et la distribution d'un produit frais, et encore moins, d'un produit aussi fragile que la banane. Mais la volonté d'agir existe et la décision de se lancer dans "I'action bananes du Nicaragua» est prise en novembre 1985. Cette opération doit viser au moins l'importation d'un wagon entier (12 à 15 tonnes) pour qu'elle soit viable.

Un intense travail de contacts avec des organisations et des mouvements tiers-mondistes, de solidarité, des organisations d'entraide, des personnes intéressées se développe. Début décembre, un comité de coordination nationale, composé de tous ces milieux, est fondé: des coordinateurs pour la Suisse alémanique et romande sont engagés. Mais le scepticisme demeure : I'opération réussira-t-elle?

Des personnes et des mouvements dans les quatre coins du pays commencent à bouger, à se mobiliser, à contacter des institutions, des restaurants, des épiceries, divers groupements, etc. Après plusieurs entretiens et discussions, assumés pour la plupart par OS3 et l'organisation «Bananenfrauen», un importateur suisse se déclare prêt à se lancer à son tour dans "l'aventure des Bananes Nica» (3). L'accord obtenu de l'importateur - première réussite de l'action - permet non seulement une introduction dans le milieu commercial mais une plus grande facilité au niveau de la distribution.

Les événements se précipitent, un formidable travail de mobilisation et d'organisation est mis sur pied, notamment par des groupes d'action (issus des groupes de solidarité avec l'Amérique centrale, des paroisses, etc.) et les Magasins du Monde. OS3 et les coordinateurs envoient des «lettres bananières» aux groupes les informant de l'action et les priant de passer des pré-commandes pour assurer l'écoulement des bananes. L'inquiétude est grande avant l'arrivée des premières commandes car il fallait atteindre au minimum les 15 tonnes fixées. A mi-mars, le chiffre des commandes se monte à 125 tonnes, ce qui représente - calculé sur la vente hebdomadaire 
habituelle - environ le $10 \%$ du marché bananier suisse. Tout le monde est étonné, les représentants du marché fruits et légumes les premiers.

\section{Les raisons d'un succès inespéré}

Nous ne pouvons, évidemment, qu'avancer des hypothèses, des suppositions difficilement vérifiables:

1. Le produit "bananes du Nicaragua» est visiblement tombé sur un terrain socio-politique favorable. Une partie au moins de la «population militante» fut ulcérée par l'étranglement économique intenté par le Goliath Etats-Unis au David Nicaragua et par les actions inqualifiables de la «Contra».

2. Il en va de même pour une partie de la population consommatrice qui, pour les mêmes raisons, a préféré l'achat de la banane Nica à celle des multinationales.

3. La vente des bananes constitue une activité concrète, palpable, de soutien au Nicaragua, souhaité par beaucoup, las des sentiers battus de la simple dénonciation.

4. L'appui des organisations d'entraide suisses à cette action lui a conféré une certaine légitimité au niveau de l'opinion publique. Ajoutons également que la "Suisse officielle», au travers du Département fédéral des affaires étrangères, avait fini par débloquer les 11 millions de francs de crédits pour les projets au Nicaragua, fonds bloqués après la réinstauration de l'état d'urgence en octobre 1985. II était donc admis officiellement que le Nicaragua n'était peut-être pas cet Etat totalitaire qu'on voulait bien nous présenter. On peut supposer que la reconnaissance officielle émise par le Gouvernement ait également contribué à dissiper d'éventuelles réticences contre le Nicaragua sur le plan politique.

5. Les milieux tiers-mondistes et écologistes, en Suisse alémanique notamment, ont mené campagne pendant de longues années contre les fruits "exotiques», invitant le public à choisir plutôt les fruits de chez nous en raison de l'exploitation des travailleurs agricoles dans le Tiers Monde et également pour lutter contre le gaspillage énergétique que représente le transport de ces fruits sur des milliers de kilomètres. Les organisateurs ont considéré tout d'abord cette situation comme un obstacle à l'action bananes. Mais une telle campagne n'a-t-elle pas été favorable à l'action Nica? En effet, la banane "alternative» permettait de faire disparaître la "culpabilité» engendrée par le fait de manger un tel fruit. La conséquence fut vraisemblablement qu'une bonne partie des personnes engagées dans la lutte tiers-mondiste "s'est ruée à cœur joie» sur cette "banane alternative».

6. Enfin, un argument plutôt commercial et valable surtout pour la Suisse romande. Les Romands, selon un grossiste engagé dans la distribution des bananes Nica, préfèrent la petite banane du type "Canaries», banane cultivée au Nicaragua, à la grande du type «Chiquita». 


\section{Les 125 tonnes sont vendues en deux jours : ne faut-il pas continuer?}

Après le formidable succès de la vente ponctuelle (4), les responsables de l'action - pouvant compter sur un importateur, deux mûrisseurs et un réseau de grossistes-distributeurs en formation - décident de poursuivre la distribution à titre d'essai pendant deux mois. Fort de ce succès commercial, l'option est prise de solliciter de plus en plus le commerce comme distributeur principal. Deux raisons guident ce choix; premièrement, dès le commencement de l'action des démarches ont été entreprises dans cette direction, deuxièmement, afin de dépasser une vente symbolique et d'assumer une vente sur une longue durée, il n'y avait pas d'autre choix que d'essayer d'implanter ce produit sur le marché suisse en passant par le commerce habituel.

Mais en plus, les organisateurs tiennent tout au long de cette action à rester fidèles aux idées de base, c'est-à-dire la recherche de relations commerciales plus équitables avec le Tiers Monde. II ne suffisait pas d'introduire ce nouveau produit sur le marché suisse, il fallait encore préserver les critères d'importation de "solidarité» que le mouvement s'était fixé, c'est-àdire de garder un surplus de solidarité d'une moyenne de 5 centimes par kilo sur le prix de vente, et ceci tout en passant par le commerce traditionnel(5),

Pour la première fois dans les annales du "commerce plus juste», l'action Nica a réussi à implanter réellement un produit sur le marché suisse en «conquérant» d'un coup environ $4 \%$ du marché, ce qui est considéré par les professionnels de la branche_comme un énorme succès vu la saturation du marché suisse et européen((6))

\section{La chaîne de magasins "Coop-Suisse" saute dans le train}

Vers la fin avril, vu le succès commercial de la Nica, la Coop-Suisse donne le feu vert à l'importation de cette banane et laise à ses coopératives régionales le soin de l'intégrer ou non à leur assortiment. Quelques coopératives régionales alémaniques, principalement dans les cantons de Berne et Zurich, décident de la mettre en vente. Feignant d'ignorer la campagne précédente, leur publicité affirme: "Après de longs efforts, nous avons enfin obtenu des bananes Nica pour la vente dans nos magasins» (7). Et contrairement à toute attente, la Nica est vendue par "Coop-Suisse» à un prix plus élevé que le nôtre. Devions-nous nous réjouir de cet événements et entreprendre des démarches pour que les autres coopératives régionales introduisent les Nica dans leur assortiment?

A première vue, la réponse semble évidente: avec "Coop-Suisse», deuxième chaîne de distribution alimentaire du pays, les ventes vont encore augmenter de manière considérable. Toutefois, la situation paraît plus complexe : nous avons réussi à créer un réseau de distribution hors des grandes chaînes, en nous appuyant notamment sur les épiceries. En prenant part à ce marché, "Coop-Suisse" risque de concurrencer ces petites épiceries et de rendre plus fragile le réseau de distribution créé par les organisateurs de 
I'action. D'autre part, par son pouvoir commercial, "Coop-Suisse» peut se passer du service de l'importateur désigné et acheter directement - par le biais de "Coop-International» - les bananes en Belgique. Le contrôle sur la filière d'importation n'existerait plus et, ce qui est plus grave, l'augmentation du prix de vente en faveur du pays producteur prélevé pour contribuer à construire peu à peu un "commerce plus équitable» pourrait être supprimé.

\section{... et les magasins Migros?}

La Direction des magasins Migros a déclaré publiquement (dans ses propres publications, reprises par un grand quotidien lausannois) et réaffirmé à la radio que la banane Nica ne correspondait pas aux exigences de qualité du marché suisse. Le démenti le plus cinglant lui est adressé justement par Coop-Suisse qui incorpore cette banane "prétendument invendable» (une banane ne peut pas dire d'elle-même qu'elle est invendable...) à son assortiment.

\section{Conclusion}

Sous peine de nous répéter un peu, nous pouvons donc affirmer qu'avec «l'action bananes du Nicaragua»:

- Nous avons réussi, pour la première fois, à introduire réellement un produit sur le marché suisse, qui y était jusqu'alors inexistant, tout en gardant un critère de politique de développement fixé par nous (surplus sur le prix de vente destiné à un projet de développement concernant les travailleurs des plantations du Nicaragua).

- Le succès commercial de notre campagne a forcé les deux plus grandes chaînes de distribution suisses à réagir (soit positivement, soit négativement).

- Enfin, il a été démontré qu'il existe en Suisse une partie non négligeable de la population qui est prête à s'engager dans une action telle que les «bananes du Nicaragua» et une autre, encore plus importante, qui est disposée, en tant que consommateurs, à participer à un commerce plus équitable avec le Tiers Monde si les conditions sont réellement réunies. Cette tranche de consommateurs est disposée à intégrer dans ses critères d'achat des valeurs telles que la justice sociale, les efforts de développement d'un pays ou la protection de l'environnement. C'est très encourageant.

A quand les «ananas alternatifs des Philippines», I'«artisanat alternatif d'Haïti», les produits "hors Botha et Pinochet» de l'Afrique du Sud et du Chili? 


\section{Notes}

1. Coopérative d'importation de produits du Tiers Monde, principal fournisseur des Magasins du Monde.

2. Pour la petite histoire: "J'étais informé de l'arrivée du premier bateau bananier partant vers l'Europe au moment où celui-ci était déjà en haute mer» nous raconta plus tard l'importateur belge.

3. L'importateur suisse avait été préalablement contacté. Outre ses doutes quant à la possibilité d'introduire la banane Nica en Suisse, il désirait prendre contact directement avec l'agence exportatrice du Nicaragua en Europe (Bananica). Celle-ci, ne disposant d'aucun réseau de distribution de commercialisation en Europe, avait chargé un importateur belge de s'occuper de la commercialisation sur le continent. Lors des premiers contacts, l'importateur suisse ne voulait pas traiter avec la firme belge ; par la suite, les deux parties ont trouvé un accord.

4. Le succès fut tel qu'une partie des 125 tonnes a dû quand même être mûrie en Belgique; la quantité dépassait les capacités des mûrisseurs suisses engagés dans l'action.

5. L'option de travailler avec le commerce traditionnel se révéla payante : de $100 \%$ des bananes commercialisées en Suisse par le réseau alternatif (OS3) durant la semaine du 10 avril, nous passions à $10 \%$ (le reste étant vendu par le commerce traditionnel) dans la première semaine de juin, ceci tout en augmentant régulièrement la vente hebdomadaire: de 27 tonnes dans la deuxième semaine d'avril à 52 tonnes hebdomadaires au début juin.

6. Il ne faut pas oublier, cependant, que pour arriver à cette situation, un immense travail de contacts et de démarchage fut à nouveau entrepris auprès de grossistes et de détaillants. Ce travail était assumé en partie par les coordinateurs et OS3, mais aussi par des membres bénévoles des groupes de leur région.

7. Publicité de la Coop-Suisse, Zurich, dans le Tages-Anzeiger du 20.4.86 